\title{
Extension of the Composite Smeared Finite Element (CSFE) to Include Lymphatic System in Modeling Mass Transport in Capillary Systems and Biological Tissue
}

\author{
M. Kojic ${ }^{1,2,3^{*}}$, M. Milosevic ${ }^{2,4}$, V. Simic ${ }^{2}$, E. J. Koay ${ }^{5}$, N. Kojic ${ }^{6}$, A. Ziemys ${ }^{1}$, M. Ferrari ${ }^{1}$ \\ ${ }^{1}$ Houston Methodist Research Institute, The Department of Nanomedicine, 6670 Bertner Ave., \\ R7 117, Houston, TX 77030, USA \\ e-mail: mkojic42@gmail.com, aziemys@houstonmethodist.org, \\ mferrari@houstonmethodist.org \\ ${ }^{2}$ Bioengineering Research and Development Center BioIRC Kragujevac, 6 Prvoslava \\ Stojanovica Street, 3400 Kragujevac, Serbia \\ e-mail: miljan.m@kg.ac.rs, vladimir.simic.991@gmail.com \\ ${ }^{3}$ Serbian Academy of Sciences and Arts, 35 Knez Mihailova Street, 11000 Belgrade, Serbia \\ ${ }^{4}$ Belgrade Metropolitan University, 63 Tadeuša Košćuška Street, 11000 Belgrade, Serbia \\ ${ }^{5}$ Department of Radiation Oncology, MD Anderson Cancer Center, Houston, TX 77030 \\ e-mail: EKoay@mdanderson.org \\ ${ }^{6}$ Center for Engineering in Medicine and Surgical Services, Massachusetts General Hospital, \\ Harvard Medical School, Boston, MA 02114 \\ e-mail: kojic@mit.edu \\ *corresponding author
}

\begin{abstract}
We have recently introduced a composite smeared finite element (CSFE) to model gradientdriven mass transport in biological tissue. The transport from capillary system is smeared in a way to transform 1D transport to a continuum, while the tissue is considered as a continuum. Coupling between the smeared pressure and concentration field is achieved through 1D connectivity elements assigned at each FE node. Here we extend our smeared model to include the lymphatic system. The lymphatic vessels are treated in a way analogous to the capillaries, by introducing the corresponding Darcy and diffusion tensors. Additional connectivity elements are added. In the numerical examples we demonstrate accuracy of the smeared model and the effects of the lymph on the pressure and concentration within the extracellular space, assuming that there is no transport to the cell space.
\end{abstract}

Keywords: Smeared finite element, convective-diffusive mass transport, biological tissue, capillary system, lymphatic system

\section{Introduction}

Mass transport from capillaries to tissue and in the reverse direction is a complex process which involves many biophysical parameters. Capillary beds are composed of capillaries of different shape, diameters and transport material parameters, as illustrated in Fig. 1. One option to model 
capillary transport of particles, molecules of nutrients, oxygen and others, is as 1D convectivediffusive process. However, due to capillary net complexity in the tissue, and particularly within tumors, it is not feasible to consider each capillary. We have recently introduced a smeared concept and formulated a composite smeared finite element (CSFE) (Kojic et al. 2017), which will be briefly described in the next section. The 1D capillary system is substituted by a continuum, with an appropriate transformation of $1 \mathrm{D}$ to $2 \mathrm{D}$ or $3 \mathrm{D}$ governing equations.

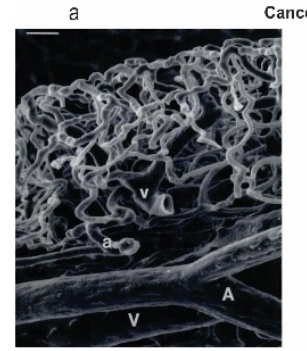

C. (S. Skinner et al. 1990)

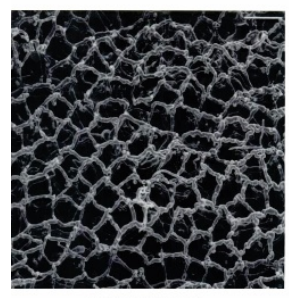

Healthy tissue
Cancerous tissue $\quad b$

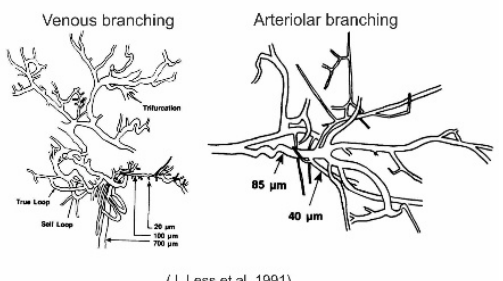

d

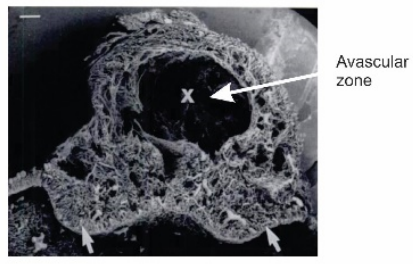

(S. Skinner et al. 1990)

Fig. 1. Capillaries within healthy (c) and cancerous tissue (a,b,d) (from Kojic et al. 2017), according to (Skinner et al. 1990, Less et al. 1991)

Besides the capillaries, there is a net of lymphatic vessels which play an important role in the living tissue, acting mainly as a drainage system. A schematic of lymph (from Google, figures) is shown in Fig. 2.

\section{Lymph Capillaries in the Tissue Spaces}

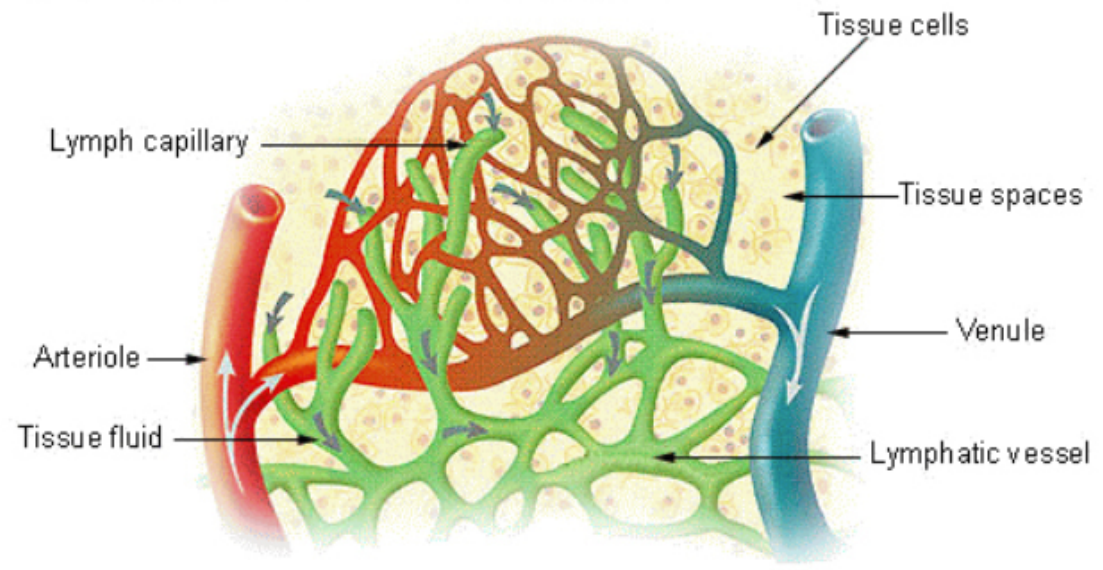

Fig. 2. Lymph capillaries within tissue (Schematic from Google) 
It is of interest in medical research and in clinical practice to have computational models which are robust and applicable, where the role of lymph can be adequately modeled. In the next section we formulate a finite element which includes the lymphatic system, starting from the previously published composite smeared finite element CSFE (Kojic et al. 2017).

\section{Fundamental relations}

In our CSFE element (Kojic et al. 2017) we have two domains, capillary and tissue domain. Now we include anther domain belonging to the lymphatic system, as shown in Fig. 3.
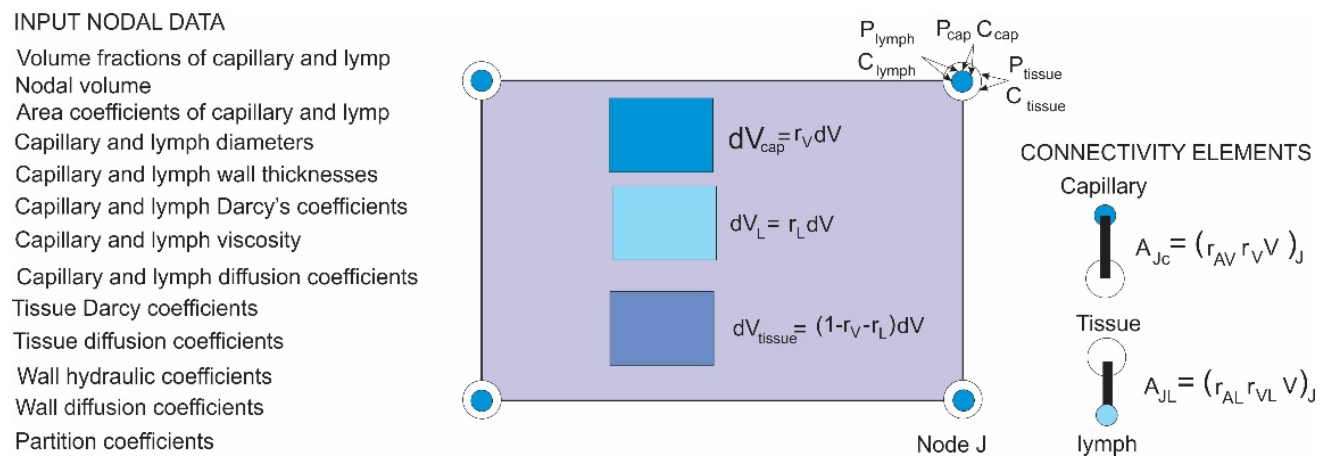

Fig. 3. Composite smeared finite element which include the lymphatic system

The volume fractions occupied by the capillaries, lymph and tissue within the finite element are:

$$
r_{V}=\frac{V_{\text {capillary }}}{V}, r_{V L}=\frac{V_{\text {lymph }}}{V}, r_{\text {Vtissue }}=1-r_{V}-r_{V L}
$$

where $V$ is total element volume. In each domain we have pressure and concentration field, therefore the nodal variables are:

$$
P_{\text {cap }}, C_{\text {cap }} ; P_{\text {lymph }}, C_{\text {lymph }} ; P_{\text {tissue }}, C_{\text {tissue }}
$$

In a way analogous to deriving the Darcy's and diffusion tensors, we can derive these tensors for the lymphatic system, therefore we have (Kojic et al. 2017):

$$
k_{D i j}=\frac{1}{A_{t o t}} \sum_{K} k_{p K} \ell_{K i} \ell_{K j}=\frac{\pi}{128 \mu_{p i p e} A_{t o t}} \sum_{K} d_{K}^{4} \ell_{K i} \ell_{K j}
$$

and:

$$
D_{i j}=\frac{1}{A_{t o t}} \sum_{K} D_{p i p e K} A_{K} \ell_{K i} \ell_{K j}
$$

where for the capillaries or lymphatic vessels: $d_{K}$ diameters, $\mu_{p i p e}$ is viscosity, $A_{K}$ internal crosssectional area of vessels, $\ell_{K i}$ directional cosines of vessels $\ell_{K i}$, and $A_{t o t}$ is total cross-sectional area of all vessels within the element: 


$$
A_{t o t}=\sum_{K} A_{K}=\frac{\pi}{4} \sum_{K} d_{K}^{2}
$$

The governing incremental-iterative equations for the fluid flow within each of the continuous domains within the element can be written as (for equilibrium iteration $i$ ):

$$
\mathbf{K}^{p} \Delta \mathbf{P}^{(i)}=-\mathbf{K}^{p} \mathbf{P}^{(i-1)}
$$

where the Darcy matrix is:

$$
K_{I J}^{P}=\int_{V} k_{D i j} N_{I, i} N_{J, j} d V
$$

and $N_{I, i}=\partial N_{I} / \partial x_{i}$ are derivatives of the interpolation functions; $\mathbf{P}$ is vector of nodal pressures. The diffusion balance equation of the finite element is (Kojic et al. 2008):

$$
\left(\frac{1}{\Delta t} \mathbf{M}^{c}+\mathbf{K}^{c}+\mathbf{K}^{c v}\right)^{(i-1)} \Delta \mathbf{C}^{(i)}=\mathbf{Q}_{c}^{e x t}+\mathbf{Q}_{c}^{V}-\frac{1}{\Delta t} \mathbf{M}^{(i-1)}\left(\mathbf{C}^{(i-1)}-\mathbf{C}^{t}\right)-\left(\mathbf{K}^{c}+\mathbf{K}^{c v}\right)^{(i-1)} \mathbf{C}^{(i-1)}
$$

where $\mathbf{C}$ is the nodal concentration vector, $\mathbf{Q}_{c}^{\text {ext }}$ is the external flux, and matrices and the source vector $\mathbf{Q}_{c}^{V}$ (evaluated at end of time step) are:

$$
M_{I J}^{c}=\int_{V} N_{I} N_{J} d V, K_{I J}^{c}=\int_{V} D_{i j} N_{I, i} N_{J, j} d V, K_{I J}^{c v}=\int_{V} v_{i} N_{I} N_{J, i} d V, Q_{c I}^{V}=\int_{V} N_{I} q d V
$$

The connectivity elements are introduced for coupling the continuous fields. They are assigned to each element node, connecting capillaries with tissue, and tissue with lymph, as shown in Fig. 3. They are fictitious 1D elements with the cross-sectional area calculated as:

$$
A_{\text {capJ }}=\left(r_{A V} r_{V} V\right)_{J}, \quad A_{\text {lymphJ }}=\left(r_{\text {AVlymph }} r_{V L} V\right)_{J}
$$

where $\mathrm{r}_{\mathrm{AV}}$ and $\mathrm{r}_{\mathrm{AVlymph}}$ are the area-to-volume ratios for capillary and lymph, respectively.

Transport through these connectivity elements depends on the hydraulic and diffusive properties of the capillary and lymph vessel walls. The governing equations have the form (6) and (8). For the fluid transport through the wall in Eq. (6) we have that the matrix for the twonode element is:

$$
K_{11}^{p}=K_{22}^{p}=-K_{12}^{p}=-K_{21}^{p}=k_{h} A
$$

where $k_{h}$ is the hydraulic coefficient, $A$ is the cross-sectional area according to Eq. (10). For the diffusion the incremental balance equation can be written as:

$$
\left(\frac{1}{\Delta t} M_{I J}+K_{I J}\right) \Delta C_{J}=-\left(K_{I J}+\frac{1}{\Delta t} M_{I J}\right) C_{J}+\frac{1}{\Delta t} M_{I J}{ }^{t} C_{J}
$$

where:

$$
M_{11}=M_{22}=\frac{1}{3} A h, \mathrm{M}_{12}=M_{21}=\frac{1}{6} A h ; K_{11}=K_{22}=-K_{21}=A D_{\text {wall }}
$$

Here, $h$ is the wall thickness, ${ }^{t} C_{J}$ is concentration at start of time step, and $D_{\text {wall }}$ is the wall diffusion coefficient. Partitioning at the wall surfaces can also be taken into account (Kojic et al. 2015). 


\section{Examples}

We have selected one example generated geometrical data available on web, and material data according to the experimental investigations within Houston Methodist Research Institute (Department for Nanomedicine, coordinator M. Ferrari), and MD Anderson Cancer Center in Houston (coordinator E. J. Koay). The purpose of the example is to demonstrate the accuracy of the smeared model with respect to the detailed FE model, and also to show the effects of the lymphatic system on convective-diffusive transport within the extracellular space.

A small tissue domain is considered, filled with cells, extracellular space, capillaries and lymph vessels. It is considered that there is no convective and diffusive transport through the domain boundary. The model consists of 5 capillaries (blue), 5 lymph vessels (red), and 70 holes representing cells, with assumption that cell transport is not present in this study (Fig. 4). The number of FE nodes in the details model is 7563, while the number of 2D FE elements in the tissue (extracellular space) domain is 6090, and there is 278 1D FE connectivity elements for the lymph vessels and capillaries. The otal area of the diffusion domain is $1521.92 \mu \mathrm{m}^{2}$ and the area of extracellular space is $691.148 \mu \mathrm{m}^{2}$. The volume fraction of capillaries is $\mathrm{r}_{\mathrm{V}}=0.0465$, while the volume fraction of lymph vessels is $r_{\mathrm{V}}=0.0249$. Cells occupy the rest of the area, which is $722.12 \mu \mathrm{m}^{2}$.
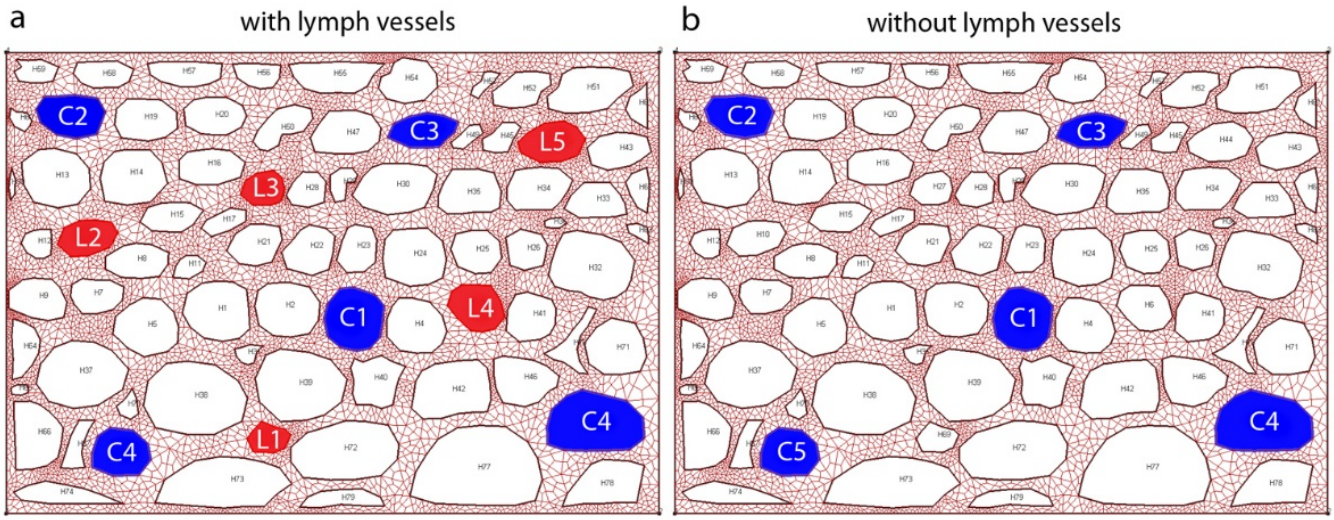

Fig. 4. Detailed model of tissue with a) capillaries (blue) and lymph vessels (red), and b) capillaries only. Holes are cells (not modeled)

We adopted in the model that transport characteristics are the same for capillary and lymph vessel walls:

- Wall Hydraulic coefficient:

$1 \mu \mathrm{m} /(\mathrm{Pa} \mathrm{s})$

- Wall Diffusion coefficient:

$1 \mu \mathrm{m} 2 / \mathrm{s}$

Diffusion coefficient in the extracellular space is $1 \mu \mathrm{m}^{2} / \mathrm{s}$, and Darcy coefficient is $1 \mu \mathrm{m}^{2} /(\mathrm{Pa} \mathrm{s})$. Prescribed values in capillaries are $\mathrm{P}=1 \mathrm{~Pa}, \mathrm{C}=1 \mathrm{M}$, while in lymph vessels we prescribed zero pressure and concentration $(\mathrm{P}=0 \mathrm{~Pa}, \mathrm{C}=0 \mathrm{M})$.

Correction function according to (Milosevic et al. 2017) is included for transport between both the capillary-tissue and lymph-tissue interfaces.

The detailed model and equivalent smeared model are generated with the following characteristics:

$100 \mathrm{FE}$ elements and $121 \mathrm{FE}$ nodes 
Capillaries (No: 5):

Mean diameter: $\quad \mathrm{D}=4.24 \mu \mathrm{m} \quad$ Volume fraction: $\mathrm{r}_{\mathrm{V}}=0.0465$

Wall thickness: $\delta=0.5 \mu \mathrm{m}$

Lymph vessels (No: 5):

Mean diameter: $\quad \mathrm{D}=3.11 \mu \mathrm{m} \quad$ Volume fraction: $\mathrm{r}_{\mathrm{V}}=0.0249$

Wall thickness: $\delta=0.5 \mu \mathrm{m}$

Extracellular space:

Volume fraction: $\mathrm{r}_{\mathrm{V}}=0.4541$

Cells:

Volume fraction: $r_{\mathrm{V}}=0.474$

It is assumed that there is no transport in the cell domain of the smeared model, which is in correspondence with the true model.

\subsection{Model with lymph system}

The pressure field in tissue of the smeared and true model is shown in Fig. 5. Mean pressure in the tissue domain of the true model is $0.54 \mathrm{~Pa}$, while it is $0.57 \mathrm{~Pa}$ in the tissue domain of the smeared model.

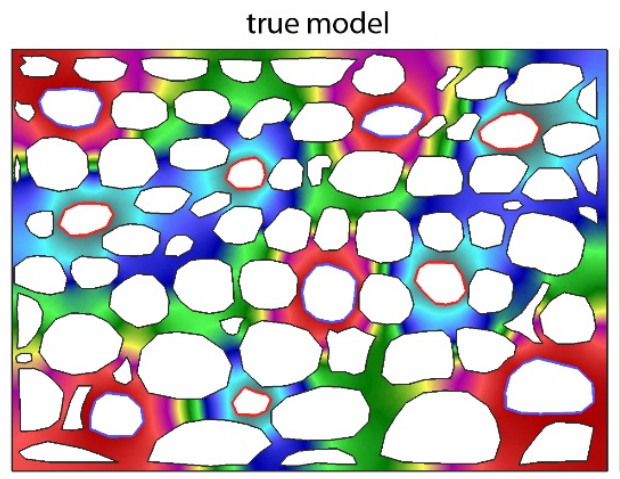

smeared model - tissue domain

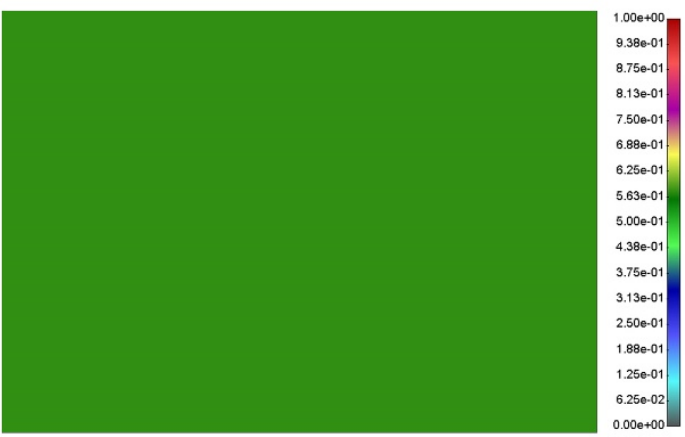

Fig. 5. Pressure field in extracellular space for true and smeared model. Since the domain with impermeable boundaries, the smeared model gives the constant field in the domain

The velocity field is shown in Fig. 6, and fluid flow is evident from the capillaries to the tissue and then to the lymph vessels. 


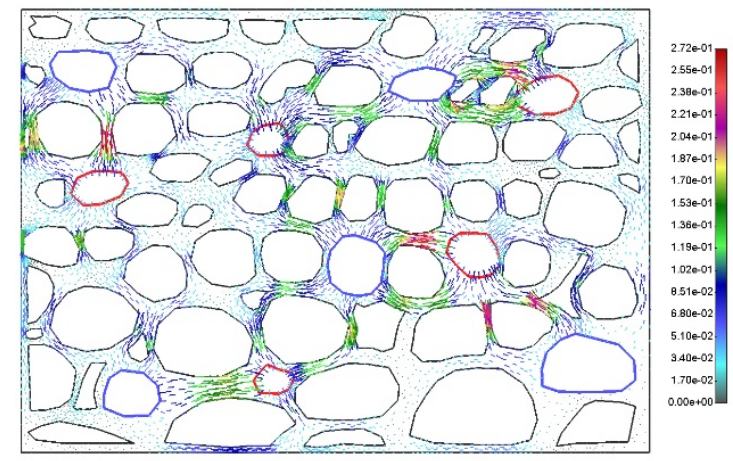

Fig. 6. Vector field of velocities in extracellular space at $t=10 \mathrm{~s}$

The concentration field in the tissue domain for both the smeared and the true model, and for two time moments is shown in Fig. 7. Mean concentration for both the models during the process can be seen in Fig. 8.

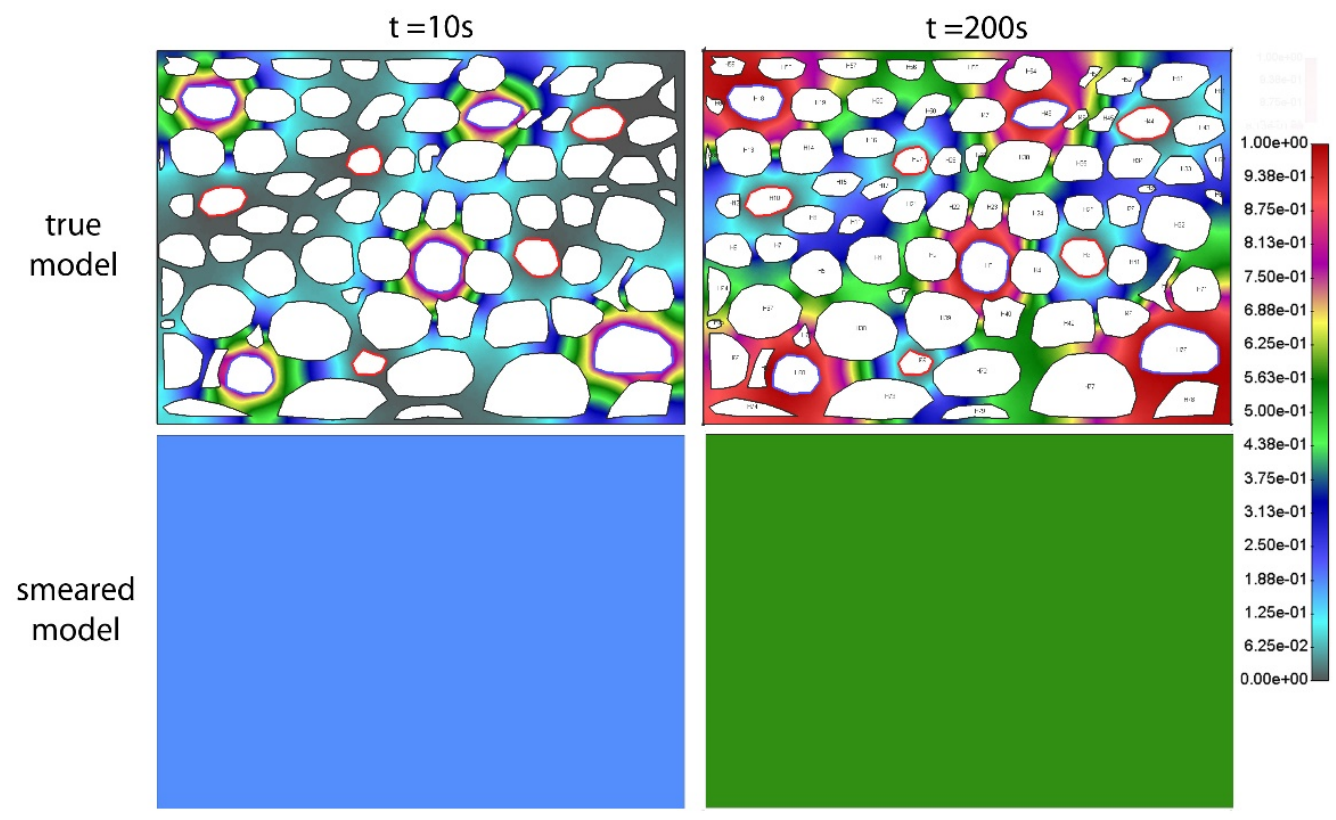

Fig. 7. Concentration in extracellular space field for true and smeared model, after $\mathrm{t}=10 \mathrm{~s}$ and $\mathrm{t}$ $=200$ s. The model with capillary and lymphatic system, with constant $c=1$ in capillaries. The domain is isolated, and concentration field is constant over the domain

We further present the evolution of the mean concentration of the detailed model and the concentration (constant over the domain) obtained by the smeared model. Regarding the prescribed concentration in capillaries, we investigated two cases: constant and bolus c(t) prescribed concentration. The smeared model gives reasonably accurate results comparing to the true model, which is shown in Fig. 8a and Fig. 8b, for both cases. 

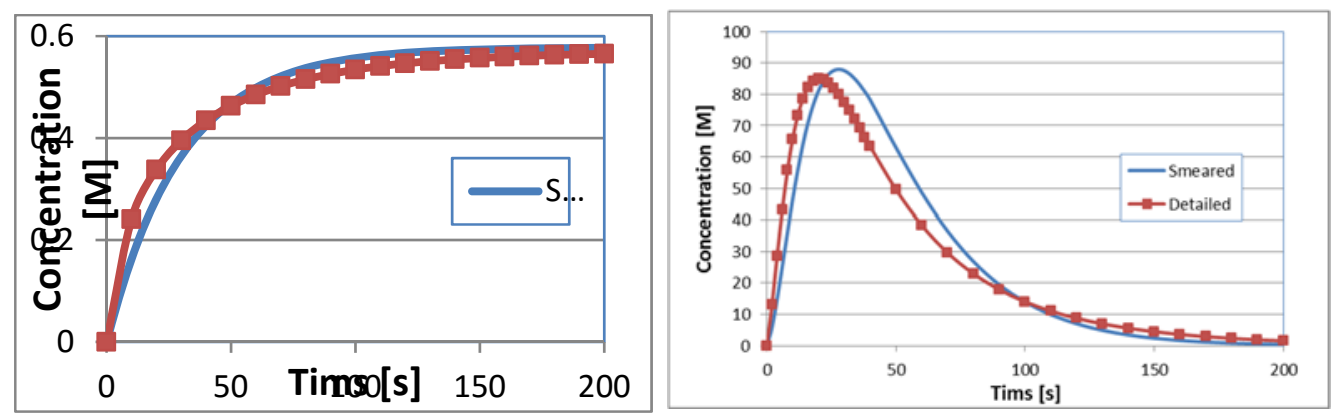

Fig. 8. Concentration vs. time in extracellular space (tissue) for true (detailed) and smeared model, with convection included, with a) constant, and b) bolus-type c(t) in capillaries.

Concentration in the true model is the mean concentration

\subsection{Model without lymph system}

For the model with capillaries only, and with $\mathrm{p}=1 \mathrm{~Pa}$, the prescribed pressure in the capillary lumen, the pressure field in tissue is constant with the same $\mathrm{p}=1 \mathrm{~Pa}$ pressure, while velocities are equal to zero. Since there are no fluid velocities, the transport of molecules within the tissue occurs due to diffusion only. The concentration fields in the tissue are shown in Fig. 9.

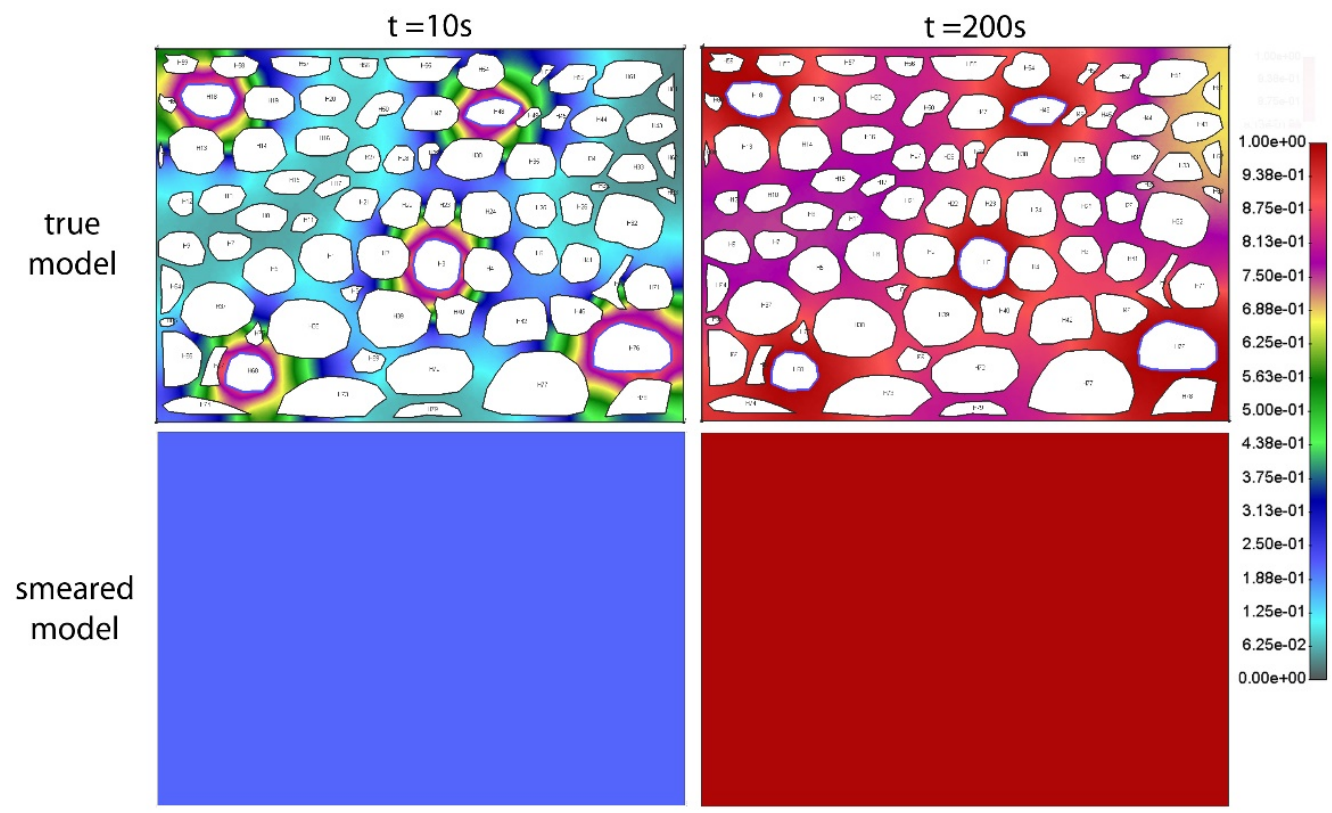

Fig. 9. Concentration field after $t=10 \mathrm{~s}$ and $\mathrm{t}=200 \mathrm{~s}$, within tissue domain of true and smeared model, without lymph vessels, and with constant $c=1$ prescribed concentration in capillaries

The evolution of the mean concentration in the tissue (true model) and concentration obtained by the smeared model is shown in Figs. 10a and Fig. 10b, for constant $c=1$ and bolus-type concentration in the capillaries. Again, a reasonable agreement between the solutions of the two models is achieved. 

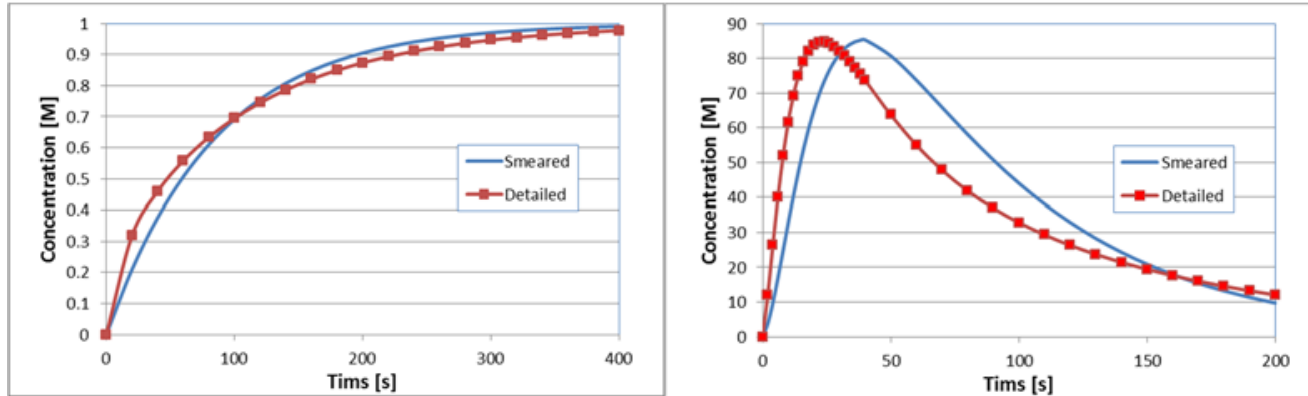

Fig. 10. Concentration vs. Time for true (detailed) and smeared model with convection included, without lymphatic system, for a) constant $\mathrm{c}(\mathrm{t})$ and $\mathrm{b}$ ) bolus $\mathrm{c}(\mathrm{t})$ concentration in capillaries

The influence of the lymphatic system can be noticed if we compare the results shown in Fig. 7 and Fig. 9, and also by comparing the diagrams given in Fig. 8 and Fig. 10. The lymphatic system acts as a sink, and in case of $c=$ const. the mean concentration is lower than in case without lymph, and the steady state is reached for approximately two times smaller time period. In case of the bolus $\mathrm{c}(\mathrm{t})$, there are slight differences when comparing the two diagrams, and in the time points when the peak is reached.

\subsection{Case with concentration and pressure gradients in tissue caused by boundary conditions}

In this example, which includes the lymphatic system, in addition to the already prescribed values at the capillaries and lymph vessels, we prescribed different pressure and concentration in the tissue at the two opposite boundaries, in order to generate gradients within the extracellular space. The prescribed values at boundaries are:

- $\quad \mathrm{c}=1 \mathrm{M}, \mathrm{p}=1 \mathrm{~Pa}$ at left vertical boundary.

- $\mathrm{c}=0 \mathrm{M}, \mathrm{p}=0 \mathrm{~Pa}$ at right vertical boundary.

Mean pressure in the tissue domain of the true model is $0.5245 \mathrm{~Pa}$, while for the smeared model it is 0.5656 . Pressure fields in the tissue domain for the true and the smeared model are shown in Fig. 11, and velocity vector field is shown in Fig. 12.
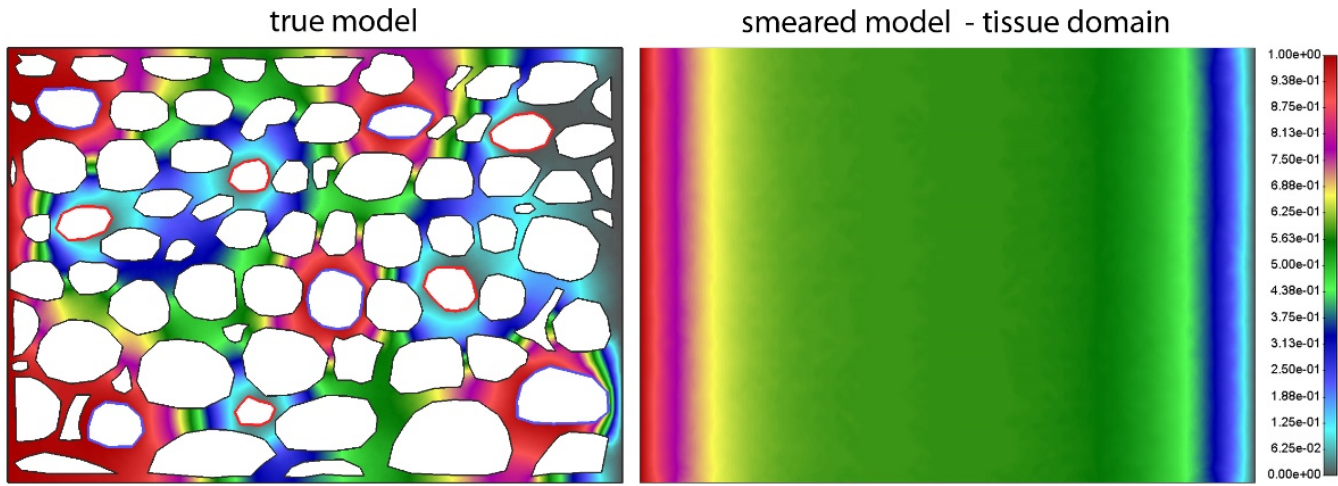

Fig. 11. Pressure field for a) detailed (true) model; b) smeared model 


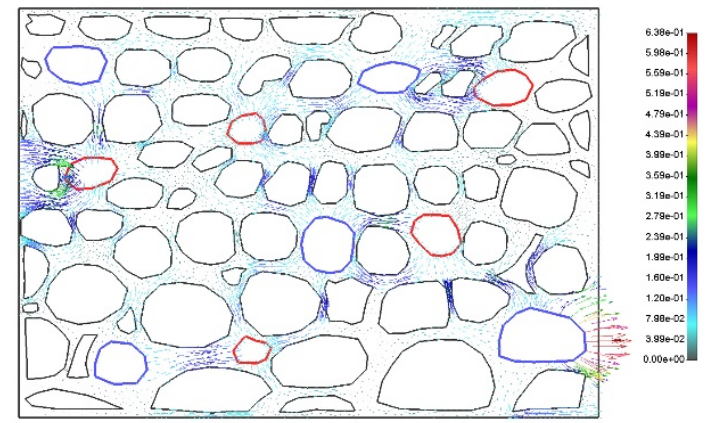

Fig. 12. Vector field of velocities at $t=10$ s, detailed model

The concentration fields within the tissue domain, in the same fashion as for the case with lymph vessels, are shown in Fig. 13.

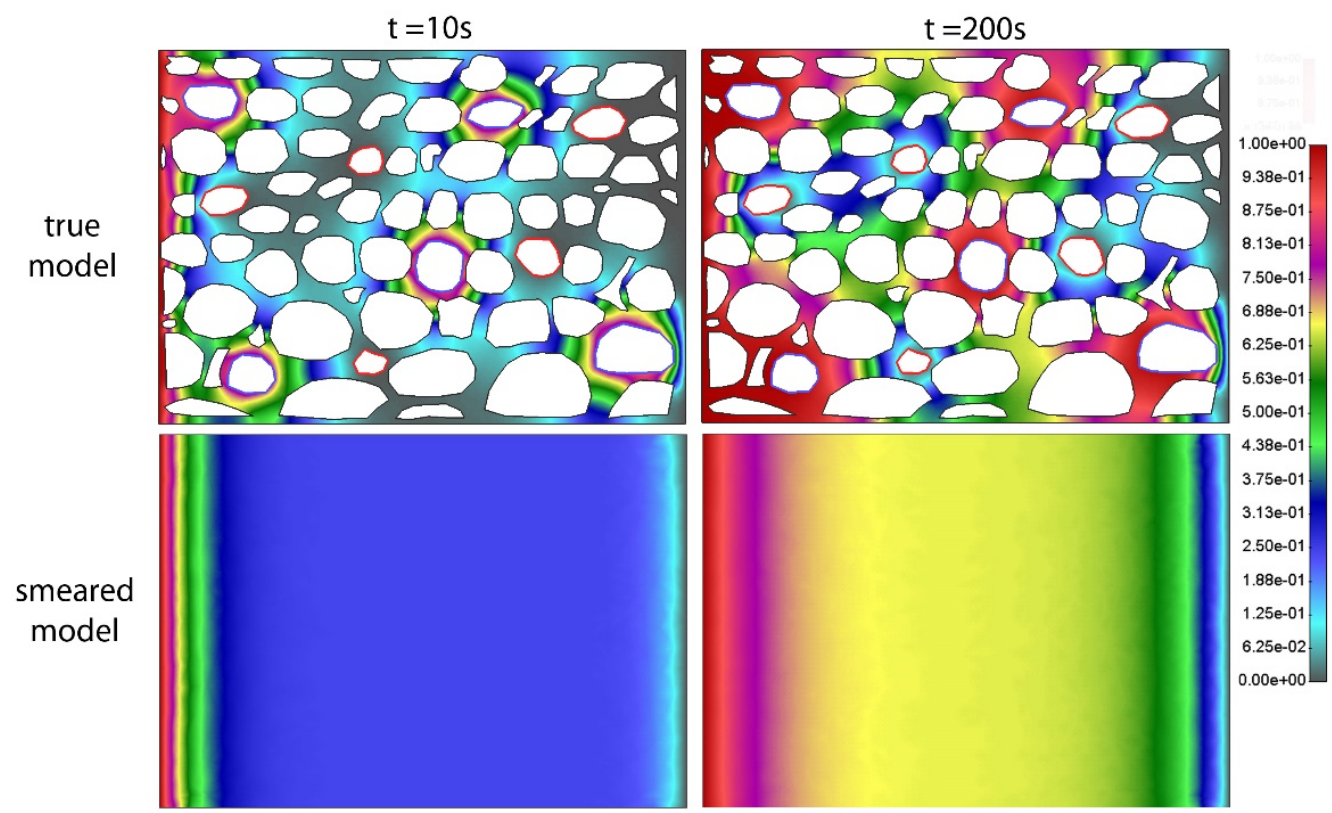

Fig. 13. Concentration field in detailed (true) and smeared model after a) $t=10 s$ and b) $200 \mathrm{~s}$ in presence of concentration gradient, for model with capillary and lymphatic system

The diagrams for Concentration vs. Time for this case are very similar to Fig. 8 (not shown here), which is a proof that values of mean concentration in the tissue domain true model during time are approximately the same as in the smeared model. The concentration fields for the model without lymph vessels, in the same fashion as for the case with lymph vessels capillaries are shown in Fig. 14. 


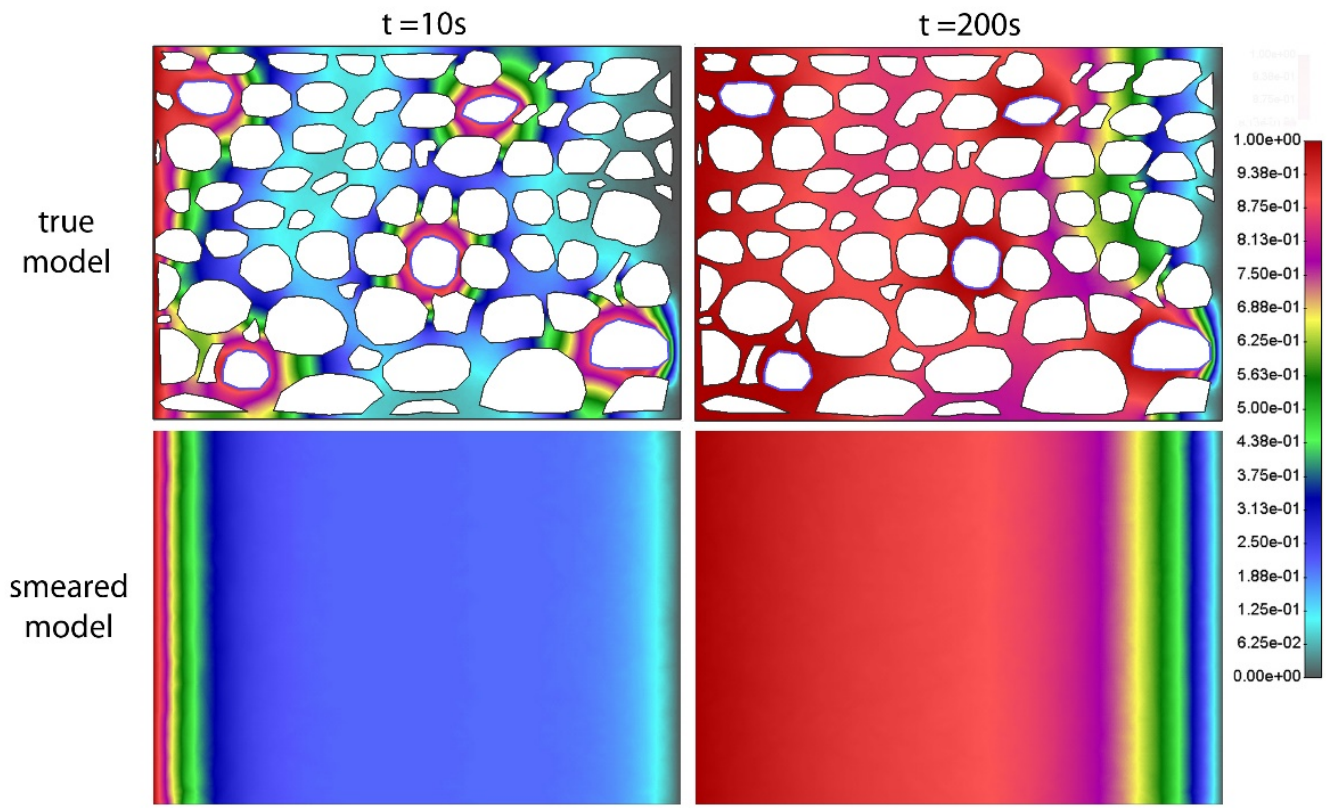

Fig. 14. Concentration field in detailed (true) and smeared model after a) $t=10 \mathrm{~s}$ and b) $200 \mathrm{~s}$ in presence of concentration gradient, for model with capillary and lymphatic system

The diagrams for Concentration vs. time for this case are very similar to Fig. 10, which is a proof that the smeared model, with a correction function included, gives a very good prediction of concentration fields, and encourage its use in models with complex geometries.

\section{Conclusions}

We have extended our previously published composite smeared finite element (CSFE) to include the lymphatic system. In numerical solutions it was shown that this extended smeared FE gives results enough accurate for practical applications in biomedical research and ultimately in medical practice. Also, the effects of the lymph on pressure and drug concentration within the extracellular space are numerically evaluated. The developed finite element, built into our FE package PAK, offer a robust tool for practical use.

Acknowledgements Dr. Ferrari acknowledges the support from NCI U54 CA210181 and The Ernest Cockrell Jr. Presidential Distinguished Chair at Houston Methodist Research Institute.

The authors acknowledge the support from the Ministry of Education, Science and Technological Development of Serbia, grants OI 174028 and III 41007, and the City of Kragujevac.

\section{References}

Kojic M, Milosevic M, Simic V, Koay EJ, Fleming JB, Nizzero S, Kojic N, Ziemys A, Ferrari M (2017). A composite smeared finite element for mass transport in capillary systems and biological tissue, Comp. Meth. Appl. Mech. Engrg., 324, 413-437. 
Kojic M, Milosevic M, Wu S, Blanco E, Ferrari M, Ziemys A (2015). Mass partitioning effects in diffusion transport, Physical Chemistry Chemical Physics, 17, 32, 20630-20635.

Kojic M, Milosevic M, Wu S, Blanco E, Ferrari M, Ziemys A (2015). Mass partitioning effects in diffusion transport, Physical Chemistry Chemical Physics, 17, 32, 20630-20635.

Kojic M, Slavkovic R, Zivkovic M, Grujovic N, Filipovic N, Milosevic M (1998, 2017). PAKFE program for structural analysis, fluid mechanics, coupled problems and biomechanics, Bioengineering R\&D Center for Bioengineering, Faculty of Technical Science, Kragujevac, Serbia.

Less JR, Skalak JR, Sevick EM, Jain EM (1991). Microvascular architecture in a mammary carcinoma: Branching patterns and vessel Dimensions, Cancer Research, 51, 265-273.

Milosevic M, Simic V, Milicevic B, Koay EJ, Ziemys A, Ferrari M, Kojic M (2017). Correction function for accuracy improvement of the Composite Smeared Finite Element for diffusive transport in biological tissue systems, Comp. Meth. Appl. Mech. Engrg., under review.

Skinner SA, Tutton PJM, O'Brien PE (1990). Microvascular architecture of experimental colon tumors in the rat, Cancer Research, 50, 2411-2417. 